\title{
The IDMIL Digital Audio Workbench: An interactive online application for teaching digital audio concepts
}

\author{
Marcelo M. Wanderley \\ IDMIL, CIRMMT, McGill University \\ Montreal, Canada \\ marcelo.wanderley@mcgill.ca
}

\author{
Travis West \\ IDMIL, CIRMMT, McGill University \\ Montreal, Canada \\ travis.west@mail.mcgill.ca
}

\author{
Josh Rohs \\ IDMIL, CIRMMT, McGill University \\ Montreal, Canada \\ j.rohs11@gmail.com
}

\author{
Eduardo A. L. Meneses \\ IDMIL, CIRMMT, McGill University \\ Montreal, Canada \\ eduardo@edumeneses.com
}

\author{
Christian Frisson \\ IDMIL, CIRMMT, McGill University \\ Montreal, Canada \\ christian.frisson@mcgill.ca
}
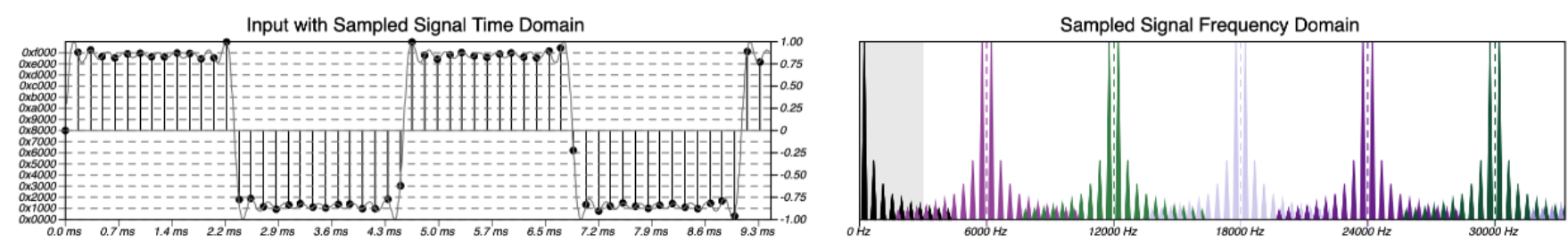

Figure 1: A view of signal sampling panels in the workbench An input signal with a fundamental frequency of $220 \mathrm{~Hz}$ and 9 (odd) harmonics is sampled with a $6 \mathrm{kHz}$ sampling rate. Left panel: Time domain. Right panel: Frequency domain. Amplitude is linear and unitless, see section3.2.1 for details

\begin{abstract}
The Input Devices and Music Interaction Laboratory (IDMIL) Digital Audio Workbench $(D A W b)$ is a web application designed for experimentation with key concepts in digital audio theory with interactive visualizations of each stage of the Analog-to-Digital Conversion (ADC) and Digital-to-Analog Conversion (DAC) processes. By experimenting with the simulation settings, numerous key concepts in digital signal theory can be illustrated, such as aliasing, quantization, critical sampling, anti-aliasing filtering and dithering. The interactive interface allows the simulation to be explored freely; users can modify parameters and examine the resulting signals visually through numerous graphs or listen to the resulting signals. The workbench has been extensively used during the 200-level Introduction to Digital Audio course at McGill University in Fall 2020.
\end{abstract}

\section{CCS CONCEPTS}

- Applied computing $\rightarrow$ Interactive learning environments; Mathematics and statistics; Sound and music computing.

Permission to make digital or hard copies of part or all of this work for personal or classroom use is granted without fee provided that copies are not made or distributed for profit or commercial advantage and that copies bear this notice and the full citation on the first page. Copyrights for third-party components of this work must be honored

For all other uses, contact the owner/author(s).

AM '21, September 01-03, 2021, Virtual

(C) 2021 Copyright held by the owner/author(s)

ACM ISBN 978-1-4503-8569-5/21/09.

https://doi.org/10.1145/3478384.3478397

\section{KEYWORDS}

sound and music computing, digital audio, digital signal processing, explorable explanations

\section{ACM Reference Format:}

Marcelo M. Wanderley, Travis West, Josh Rohs, Eduardo A. L. Meneses, and Christian Frisson. 2021. The IDMIL Digital Audio Workbench: An interactive online application for teaching digital audio concepts. In $A M$ '21: Proceedings of the 16th International Conference on Audio Mostly, September 01-03, 2021, Virtual. ACM, New York, NY, USA, 8 pages. https://doi.org/10. $1145 / 3478384.3478397$

\section{INTRODUCTION}

Digital audio is ubiquitous in our daily lives. Digital audio techniques allow individuals to communicate verbally (by phone), consume media such as podcasts, audio recordings, and digital video, and distribute and listen to music, which are common daily activities for millions of people. Converting acoustic waves (sounds) to their digital representation and back is, therefore, an essential step in any of the above activities. Though the main mathematical concepts allowing for analog-to-digital conversion have existed since the 1920's, digital devices became more popular, at least in the case of music, around the end of the 70's thanks to advances in technology such as the compact disc player and, later on, personal computer audio.

For music technologists and audio designers, it is, therefore, essential to clearly understand the process of creating digital representations of analog sound signals and, conversely, of obtaining analog signals back from their digital representations. Furthermore, given that this conversion is not perfect (e.g. it is impossible to completely eliminate quantization errors), the limitations of these 
processes must be taken into account when designing or choosing digital audio applications for sound acquisition and reproduction.

Though several excellent resources exist explaining digital audio concepts in various levels of detail, including textbooks, video tutorials and code examples, the most intuitive way to fully grasp these concepts would be a hands-on, interactive approach. Looking at and listening to signals generated by devices or recorded by a microphone and witnessing the conversion process while changing acquisition and reproduction parameters is a compelling way to understand digital audio concepts and the effects of these parameter changes.

The purpose of the DAWb is to illustrate key concepts in digital audio theory with interactive visualizations of each stage of the conversion processes by simulating the processes that would be occurring in actual ADC and DAC. By experimenting with the simulation settings, numerous key concepts in digital signal theory can be illustrated, such as aliasing, quantization, critical sampling, anti-aliasing filtering and dithering. The interactive interface allows the simulation to be explored freely; users can examine the signals both visually through numerous graphs or by listening to the resulting signals directly ${ }^{1}$.

\section{RELATED WORK}

Several resources exist for teaching and learning Digital Audio (DA). In this section, we review 1) books on DA, which are generally non-interactive, but sometimes provide interactive examples as supplementary materials (section 2.1); 2) explorable explanations, emerging in STEAM fields (Science, Technology, Engineering, Arts and Mathematics), that co-locate textual explanations and interactive examples (section 2.2); 3) Online simulations covering scientific concepts, and 4) recent examples of interactive course materials for teaching DA (section 2.4).

\subsection{Books on Digital Audio}

Books on DA generally separate non-interactive text and figures located in the main document (the book) from additional examples made available as supplementary materials.

Many DA and Digital Signal Processing (DSP) books introduce DA concepts either from an engineering or a mathematical perspective. Some of the widely used textbooks in this field include Principles of Digital Audio [10], The Art of Digital Audio [13], DSP First [8] and A Digital Signal Processing Primer [11].

Pohlmann's Principles of Digital Audio and Watkinson's The Art of Digital Audio are reference textbooks for introductory music technology classes, which present a wealth of technical information related to DA devices and systems. McClellan et al.'s DSP First and Steiglitz's A Digital Signal Processing Primer take a mathematical approach to DA and are typically used in higher-level undergraduate music technology courses, though with many sections very useful for introductory courses.

Pohlmann's chapter 2 deals with the fundamental concepts of DA. Figures 2.2 and 2.7 describe the processes of sampling and quantization respectively, and are excellent examples of how DA concepts can be statically illustrated to students. Though these

\footnotetext{
${ }^{1}$ The tool can be accessed at https://idmil.gitlab.io/course-materials/mumt203 interactive-demos/
}

figures are very helpful, students often have trouble generalizing use cases from parameters captured in printed illustrations to other parameter sets. Though some books such as Ballora's Digital Audio and Acoustics for the Creative Arts [3] do provide figures showing multiple parameter values, for instance, figures 5.3 and 5.9, these are still limited to only a couple values per figure. Common questions that arise in class when these figures are discussed include "What happens at exactly half the sampling rate?", "What happens when the input signal is slightly above half the sampling rate?", "When do quantization artefacts become audible?", which are not straightforward to answer using static figures.

Books such as McClellan et al.'s DSP First and Dutoit and Marqués' Applied Signal Processing [5] feature extensive examples of DSP exercises with MATLAB, but with a high entry barrier: students typically need to purchase licenses or get limited tokenized access that may be provided by universities, to install the software along with many additional libraries, and to learn how to get started to launch examples. DSP First also provides excellent videos of animations from MATLAB simulations, though parameters are fixed, rendering the simulations non-interactive.

The inspiration for the DAWb resulted from our need to provide a dynamic illustration of the sampling and quantization effects in simulated signals, i.e. to make these static figures dynamic by allowing the user to choose the input signal type, frequency, amplitude and phase, the sampling frequency and the number of bits used for quantization. Furthermore, for such a tool to be useful for many, it should be widely and freely available, not dependent on proprietary frameworks, and as simple to use as possible.

\subsection{Explorable Explanations in STEAM Fields}

Explorable explanations, emerging in STEAM fields, combine text and examples into interactive materials, co-locating theoretical and practical information. The concept of explorable explanations has been envisioned by Bret Victor, who proposed the following ideas: 1) active readers should be able to play with authors' assumptions and analyses in reactive documents that display the consequences of their active reading; 2) explorable examples should make abstract concepts concrete and allow readers to develop an intuition for how a system works; and 3) contextual information should allow readers to learn related material just-in-time, and to cross-check authors' claims [12]. The reader is invited to interact with explorable explanations from the many examples across STEAM fields presented in the hub curated by Nicky Case and other contributors [4].

DAWb inherits the concept of explorable explanations and contributes one first step towards interactive materials for DA: an explorable graph which illustrates DA concepts such as aliasing and quantization, in order to make these concepts easier to understand.

\subsection{Online Simulations}

An excellent example of the use of interactive online simulations is the Physics Education Technology Project (PhET) at the University of Colorado, developed since the early 2000s [9]. PhET is a collection of dozens of freely available simulations covering concepts in physics, chemistry, mathematics and other fields ${ }^{2}$.

\footnotetext{
${ }^{2}$ https://phet.colorado.edu/en/about
} 

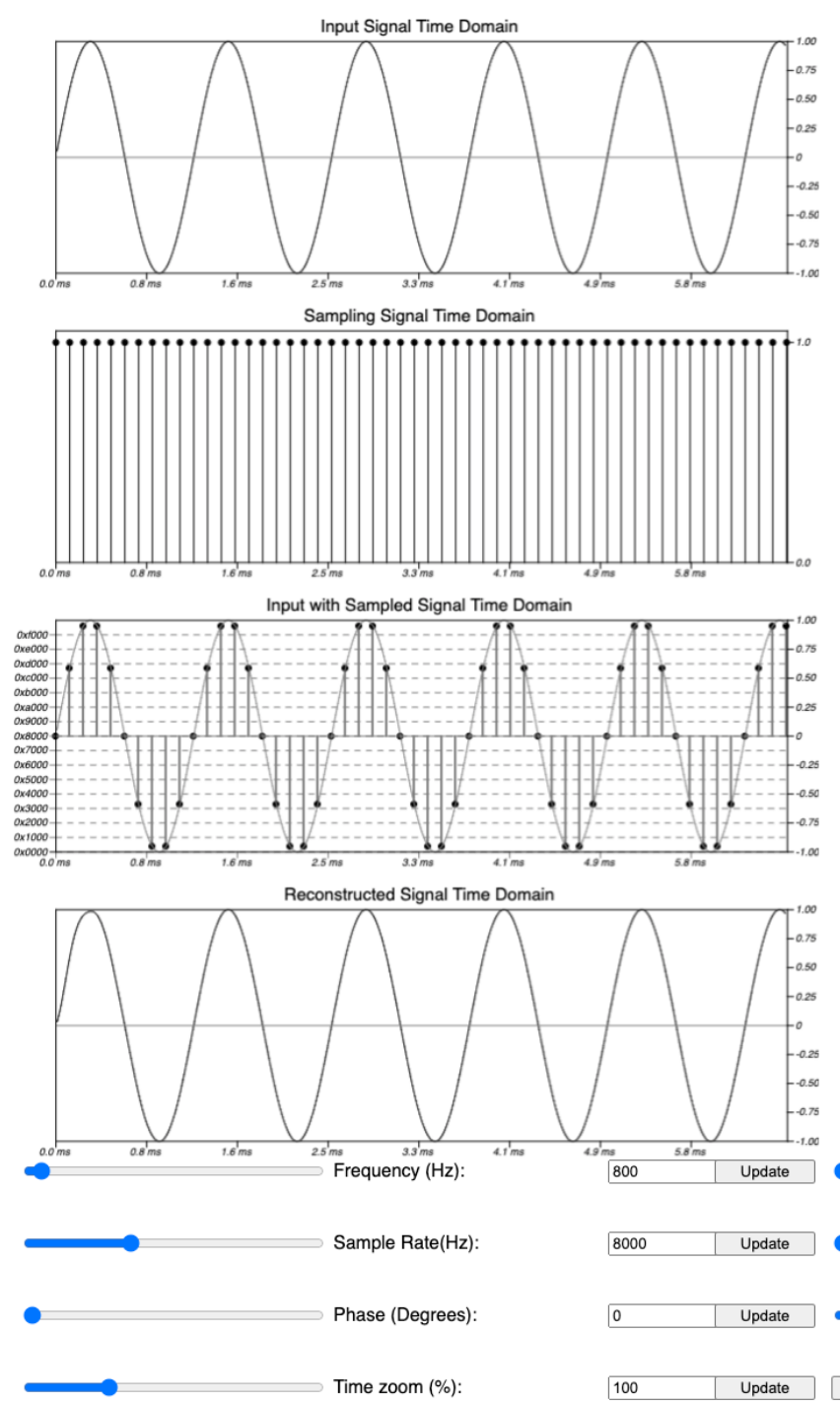

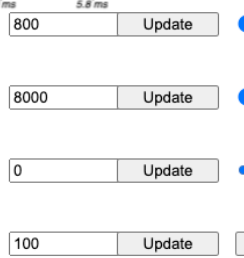

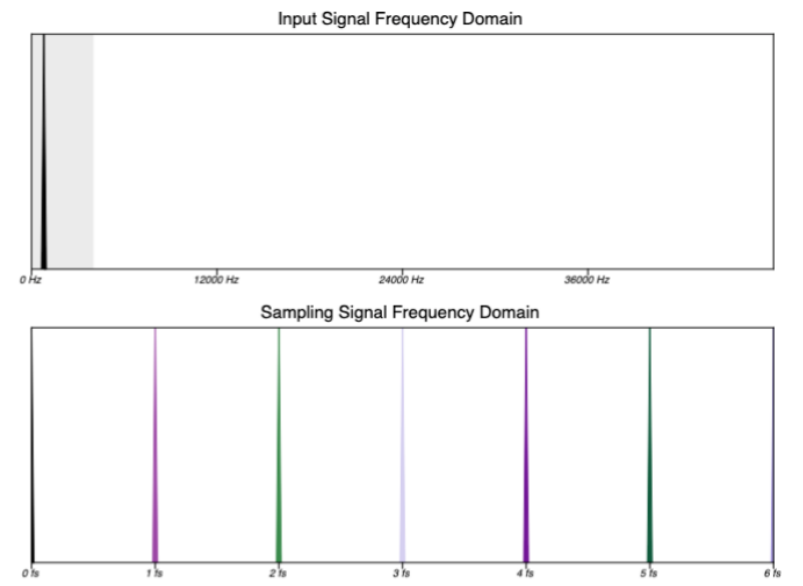
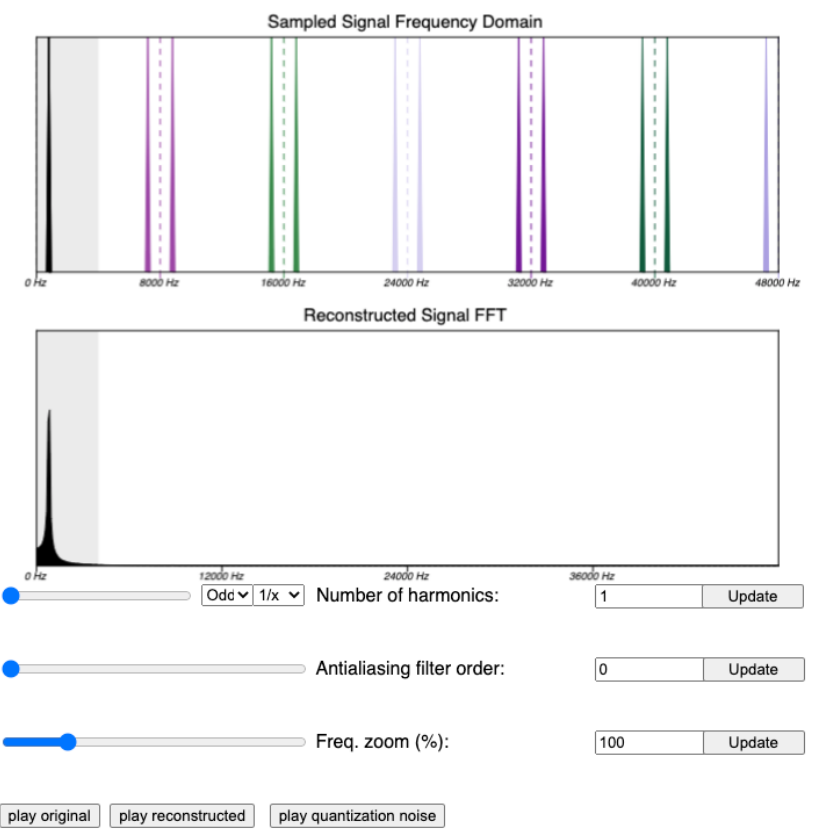

Figure 2: The Sampling \& Aliasing view. Left panels: Time domain. Right panels: Frequency domain. Panels, top to bottom: input signal, sampling signal, sampled signal and reconstructed signal. Bottom of image: control sliders for frequency selection, zoom, etc. and buttons to allow for audio display of original and reconstructed signals, as well as quantization noise.

An important component of the PhET project is the effort to validate their approach. Research on the impact of the simulations in the PhET project has shown that simulations are an engaging and effective educational tool when students' interaction with the simulations are directed by their own questioning [1]. Furthermore, Wieman et al. indicate that "students have little fear of breaking simulations or hurting themselves" and "were observed to do more spontaneous experiments with the simulation than with the corresponding real electrical equipment" [15]. PhET simulations and $\mathrm{DAWb}$ are similar in that they allow for unrestricted interaction with simulation parameters and can be useful to replace laboratory equipment in class demonstrations. Major differences between them are the absence of a game-like environment in DAWb and its complexity compared to mostly single concept animations in PhET.

\subsection{Interactive Materials on Digital Audio}

Several examples of interactive materials for teaching DA concepts have been recently proposed.

Alisson et al. developed web-based examples for a summer outreach camp designed to teach computer programming concepts to a young audience through the activity of music-making [2]. Building upon a subset of the same technologies and libraries (Gibber, NexusUI.js, Tone.js), Xambó et al. proposed an elective master course on DA [16]. While the course aims at teaching Web Audio technologies to students, the course materials themselves were not 
implemented with Web Audio technologies but rather typeset with LaTeX and rendered as PDF slides, with code examples separated.

Kristis et al. developed iMuSciCA to introduce secondary school students to STEM subjects by engaging them in music activities. The most similar component to DAWb implemented in the iMuSciCA platform is named " $2 \mathrm{D}$ visualizations" and displays audio signals in 3 HTML canvases: the audio waveform in the time domain, the amplitude of the different frequency harmonics of the input signal, and the frequencies over time (2D spectrogram) [7]. Parameters of these components are not, however, exposed through interactive widgets.

Peters et al. released trackswitch.js, a web-based audio player enabling researchers to present examples and results from scientific audio processing applications such as sound source separation, by letting readers switch between multiple audio tracks, while synchronously indicating the playback position within images associated with the audio tracks [14]. DAWb and trackswitch.js share one goal of exposing the contributions of different nodes and sources in the audio signal processing pipeline. However, trackswich.js requires visual representations to be pre-computed as bitmap images, while DAWb computes visual representations in real-time, allowing users access to various visual layouts in order to access and examine all different elements structuring the graphs.

Frisson et el. used web-based and Web Audio technologies as a proxy to teach haptic illusions for the WebAudioHaptics tutorial, with interactive audio examples embedded in course materials [6]. While WebAudioHaptics and DAWb share one goal of using web-based and Web Audio technologies to create explorable explanations with DA that are embeddable in course materials, they employ different approaches for authoring: WebAudioHaptics interactive illustrations are authored through visual programming with PureData patches rendered with WebAudio using WebPd with parameters exposed through NexusUI.js widgets, while DAWb is authored through scripted programming using p5.js. With DAWb, we needed more in-depth access to samples for both signal generation and visualization, which is easier to achieve with scripted programming.

These works illustrate the following challenges: co-locating interactive examples and course materials, exposing parameters of interactive examples, increasing accessibility limited by visual representations, authoring interactive materials with real-time audiovisual feedback. All of these challenges are dependent on and constrained by the choice of web-based and Web Audio technologies to author and render interactive course materials for DA.

\section{DESIGN}

The DAWb aims to provide an accessible interactive platform for intuitively exploring key concepts in DA. Both Time- and Frequencydomain views of signals are presented, as well as audio rendering. Numerous modular graphs (panels) are available and can be used to illustrate DA concepts. To provide a coherent view of individual concepts, three views are proposed:

- sampling \& aliasing

- quantization \& dither

- all panels and sliders
The first two focus on sampling and quantization and their effects, respectively, allowing students to concentrate on each issue separately by reducing the number of panels available to the number necessary to understand each concept independently. The third view presents a collection of all panels and is useful for more advanced students or developers, allowing them to experiment with more complex parameter manipulations.

The DAWb is currently an interactive visual explanation that includes audio playback, developed with web-based and Web Audio technologies, with the intention of being embedded in web-based course materials.

\subsection{Usage Examples}

In this section, we illustrate the use of the DAWb sampling and quantization views with four examples.

Figure 1 shows part of the sampling view where an input signal composed of 10 partials-a fundamental frequency of $220 \mathrm{~Hz}$ and its first 9 odd harmonics-is sampled with a sampling frequency of $6 \mathrm{kHz}$. Given that the input signal's maximal frequency is 4.18 $\mathrm{kHz}$ which is higher than the Nyquist frequency of $3 \mathrm{kHz}$ (i.e. half the sampling frequency), aliasing occurs. In the gray region, this can be seen as the three purple frequencies $(1.82 \mathrm{kHz}, 2.26 \mathrm{kHz}$ and $2.7 \mathrm{kHz}$ ), which are images of the input signal around the sampling frequency.

In figure 2, an $800 \mathrm{~Hz}$ input sinusoidal signal is sampled with a sampling frequency of $8 \mathrm{kHz}$, yielding 10 samples per period of the input signal (cf. 3rd panel from the top, left side). The reconstructed signal, in this case, has a frequency of $800 \mathrm{~Hz}$, as with the input signal. On the right side, the top and bottom panels show a gray region starting at $0 \mathrm{~Hz}$ that extends to half the sampling frequency (aka the Nyquist frequency), indicating the region of interest in the sampling process. The second panel illustrates the fact that the frequency domain representation of an impulse train in the time domain is another impulse train with frequencies at multiples of the sampling frequency. The third panel on the left shows the result of multiplying a time-domain input signal by a sampling signal: the appearance of images (copies) of the input signal frequencies around multiples of the sampling frequency. These are the frequencies that eventually become aliases when parameters are chosen so that the sampling theorem is not respected.

In figure 3, a $440 \mathrm{~Hz}$ signal is sampled with a $48 \mathrm{kHz}$ sampling signal. In this case, no aliasing takes place, as the sampling frequency is much higher than twice the signal frequency. The choice of a low bit depth (3 bits) leads to a reduced number of quantization steps available, in this case, 8 steps, yielding large errors between the actual sample amplitudes and the closest available quantization step, as seen in the bottom panel that displays the difference between the signals in the two panels above. This error, known as quantization noise, can be seen as extra frequency components in the second and third panels in the right and can be heard in both the reconstructed signal and the quantization noise when pushing the corresponding buttons.

Finally, figure 4 shows the same parameters as in Figure 3, but this time a small amount of dither noise is used in the acquisition process. Note that in the time-domain panels, the quantized amplitudes became less symmetrical due to the added noise. In the frequency 

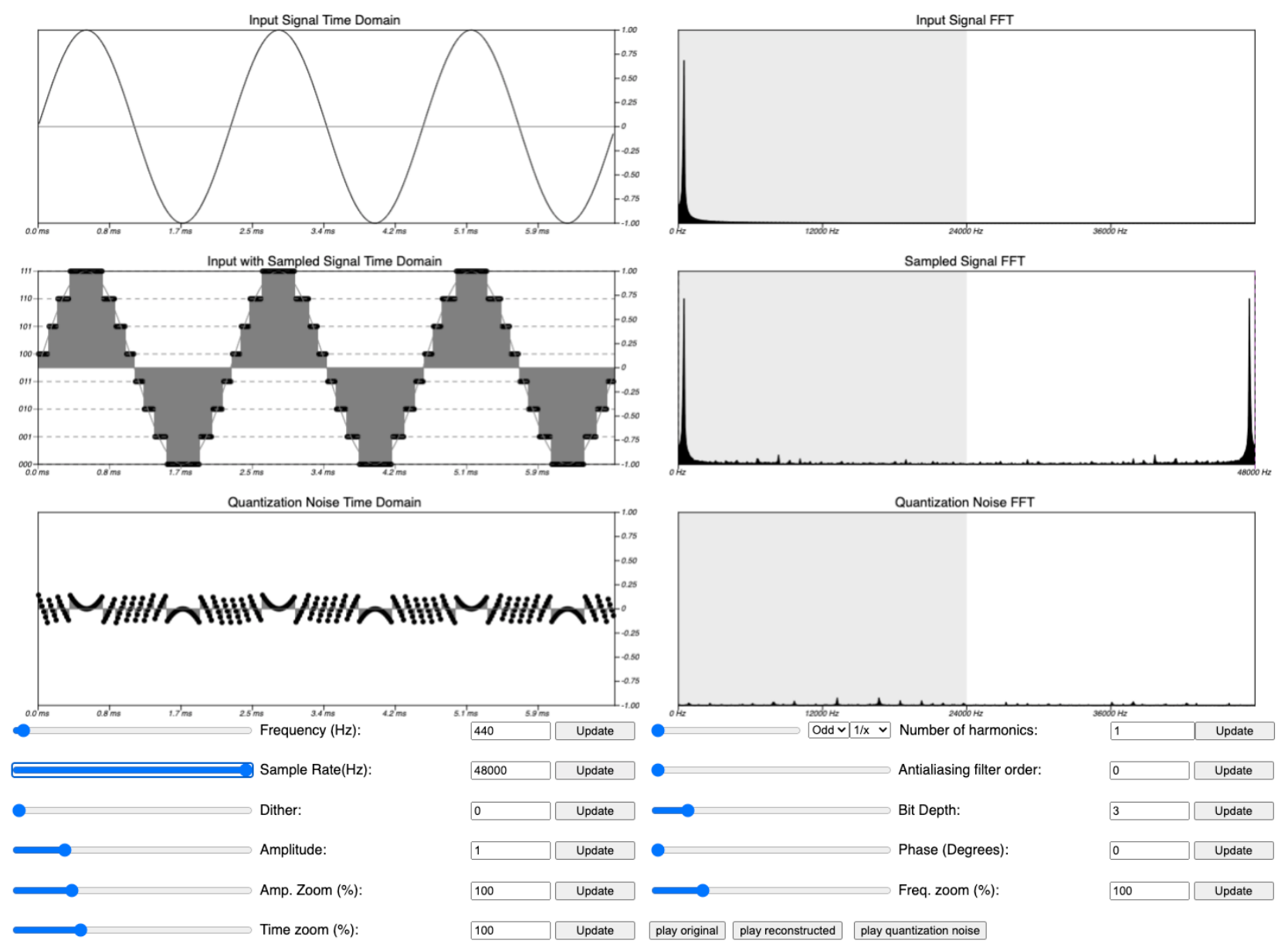

Figure 3: The Quantization and Dither view. Left panels: Time domain. Right panels: Frequency domain. Panels, top to bottom: input signal, quantized signal and quantization noise. Bottom of image: control sliders for frequency selection, amount of dither, zoom, etc. and buttons to allow for audio display of original and reconstructed signals, as well as quantization noise.

domain, the various small frequency peaks which appeared in the previous figure were removed, though at the expense of an increased noise floor.

\subsection{Implementation Details}

The DAWb is implemented using the web platform, allowing anyone to easily view the interactive demonstrations without having to download or install anything other than their web browser. The implementation of the simulation, drawing, and interactive widgets are achieved primarily using plain JavaScript. The tool is open source under an MIT license ${ }^{3}$. Though the tool was tested using Chromium-based browsers, individuals using the DAWb in Firefox have not reported any compatibility issues as of the time of this writing. More comprehensive testing remains for future work.

3.2.1 Simulating the Conversion Processes. As our demonstration takes place purely in the digital domain, we must simulate the

${ }^{3}$ The source code can be accessed at http://www-new.idmil.org/project/the-digitalaudio-workbench/
ADC-DAC processes in the discrete-time domain. The typically continuous and infinite-resolution analog input and output signals are approximated as discrete-time signals.

The ADC process consists of several steps, including anti-aliasing, sampling, and quantization. All of these are simulated in our model: anti-aliasing is achieved with a windowed sinc Finite Impulse Response (FIR) low-pass filter of order specified by the user; sampling is approximated by downsampling the input signal by an integer factor; and quantization is achieved by multiplying the sampled signal by an integer based on the user-requested bit-depth, and then rounding every sample to the nearest integer. The DAC process is simulated by zero-padding and low-pass filtering the sampled and quantized output of the ADC simulation.

In summary, the continuous-time input is simulated by a $96 \mathrm{kHz}$ discrete-time signal, the sampled output of the ADC process is simulated by a downsampled and quantized signal, and the continuoustime reconstruction, output by the DAC, is simulated by upsampling the "sampled" signal back to $96 \mathrm{kHz}$, the maximum sampling rate supported by WebAudio at the time of writing. 

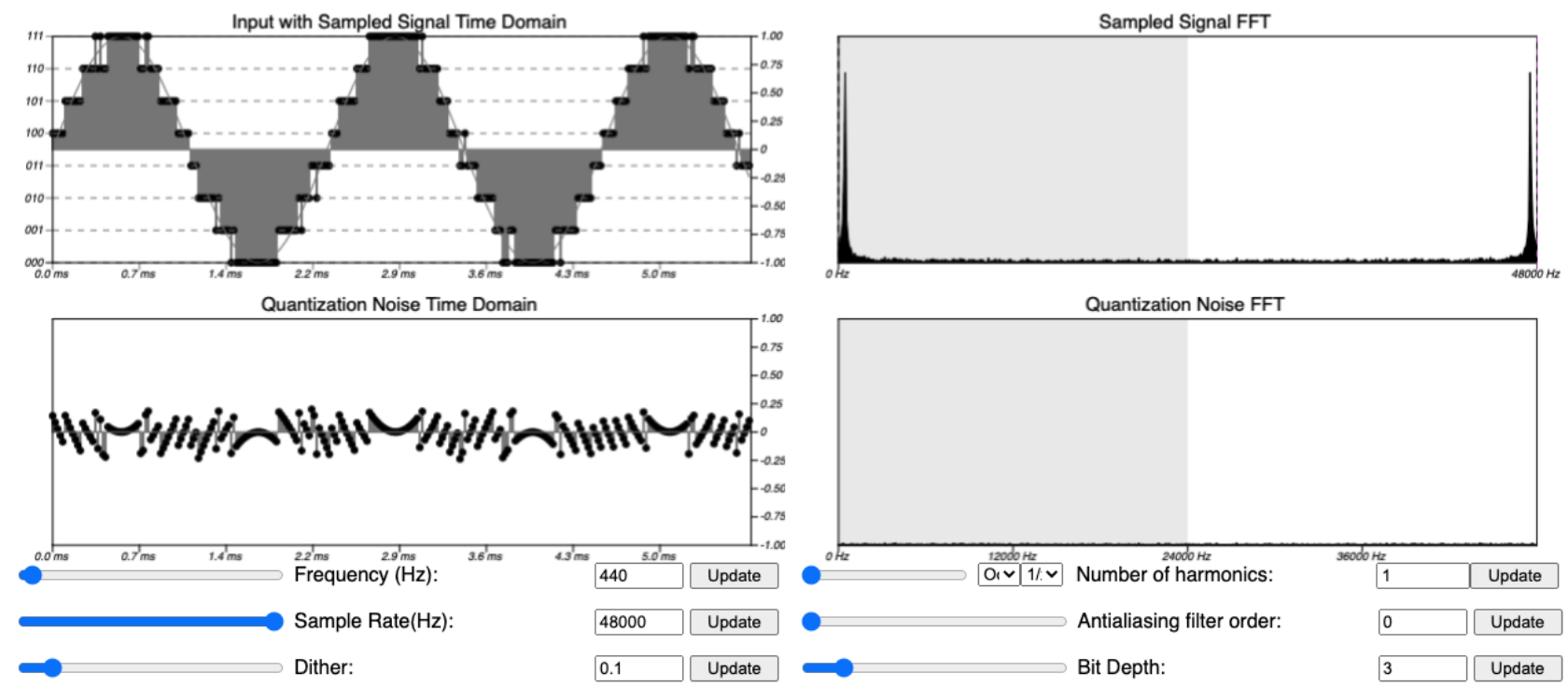

Figure 4: Example of dithering (excerpt). Note that the various small frequency peaks which appeared in the previous figure were removed.

Since the entire simulation takes place in the digital domain, the vertical amplitude dimension of the graphs are unitless values, from -1 to 1 for time domain graphs and from 0 to 1 for frequency magnitude graphs. For this reason units are not applied to the amplitude labels on the graphs. No logarithmic adjustments are made to frequency nor amplitude axes, although this may be added in the future.

For more details, the reader is encouraged to peruse the source code. Many comments have been included to aid readers who are unfamiliar with JavaScript. While the best resource for definitive answers to questions regarding the implementation of the simulation is the source code itself, readers are also invited to contact the authors with any questions.

3.2.2 Interactive Parameters. At the time of writing, the simulation uses a simple additive synthesis algorithm to produce a test input. The user can control the fundamental frequency of the signal, the number of harmonics (from 1, a cosine wave, to 20), the initial phase, and the peak amplitude of the signal. The frequency and number of harmonics are limited to allow aliasing to be demonstrated without introducing aliasing in the analog approximation at $96 \mathrm{kHz}$. The user can also select whether to generate only odd or only even harmonics, control the amplitude roll-off of harmonics, allowing the generation of a triangle, square, and other more unusual waveforms.

While controlling the parameters of the input signal is useful, the most important parameters presented by the DAWb are those that control the simulation. The bit depth can be selected from 1-bit to 16-bit. The peak amplitude of the dithering noise can be set from 0.0 to 1.0. The FIR filter order of the input anti-aliasing filter can be set from 0 (no anti-aliasing) to 200. Finally, the sample rate of the $\mathrm{ADC}$ process can be set from $3 \mathrm{kHz}$ to $48 \mathrm{kHz}$. In addition, sliders are also provided for users to zoom in and out for both time and frequency.
Although a continuous slider is presented to the user for changing the sampling rate, the sampling rate is actually internally set by an integer resampling factor. This makes the resampling procedures in the simulation very simple to implement: with a resampling factor $N$, downsampling is achieved by keeping only every $N$ th sample and discarding the rest, while upsampling is achieved by stuffing $N-1$ zeros after every sample and then low pass filtering with an order 200 FIR sinc filter. The actual sampling rate of the simulation is displayed in the user interface to avoid confusion.

3.2.3 Drawing the Graphs. Based on the user-selected parameters, the simulation generates an input signal, a sampled signal, and a reconstructed signal, as well as the spectra of these three signals using the FFT. Apart from directly drawing these signals, additional information, as well as entire graphs, can be generated analytically by inspecting the simulation parameters.

Among the many figures that are currently implemented, there are time-domain graphs of the signal at various stages through simulation, FFT-based magnitude graphs of the same, graphs overlaying multiple time-domain signals (e.g. figure 5), and several analytic frequency-domain figures (e.g. figure 6).

The analytic frequency-domain figures are generated based on the parameters of the simulation (e.g. fundamental frequency and number of harmonics of the input signal), and do not rely on the FFT. Apart from avoiding distortions inherent in the FFT such as windowing artifacts, this allows additional information about the signal to be encoded in the figure. For instance, changing the color of the aliased images at multiples of the sampling rate helps visualize which images are aliased back to the region of interest (in gray, in the figure). Similarly, information such as the pass-band of the reconstruction filter, the amplitude of low-bit-depth quantized steps, and the time- and frequency-domain representations 
of the sampling signal can all be determined analytically from the parameters of the simulation.

Input (solid), Sampled (lollipop), Reconstructed (dotted), Time Domain

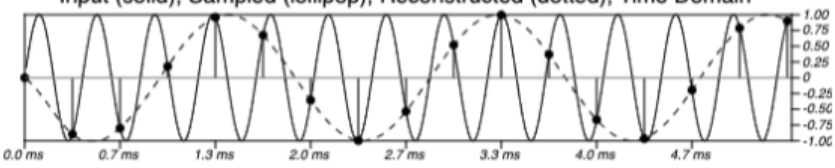

Figure 5: Time-domain signals overlaid for comparison. Note the resulting aliased signal as there were less than 2 samples taken per period of the input signal (i.e. the input frequency was higher than the Nyquist frequency).

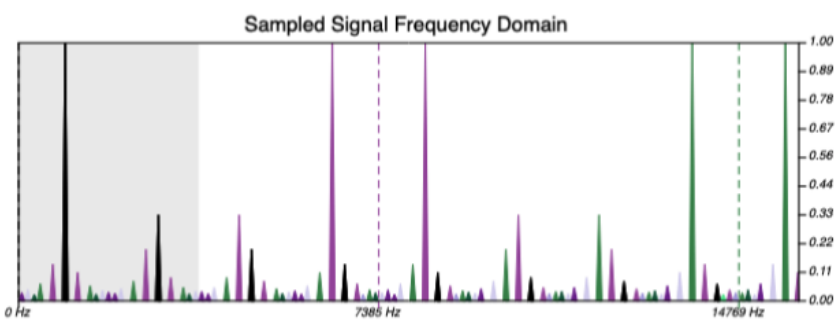

Figure 6: Frequency content of an input signal (fundamental frequency $1 \mathrm{kHz}$ and 19 harmonics) sampled at $7385 \mathrm{~Hz}$. The gray region of the graph (from $0 \mathrm{~Hz}$ to the Nyquist frequency) shows the two first partials of the signal (in black) together with several aliased images of the input at multiples of the sampling rate (in color).

\section{USAGE IN CLASS}

The Introduction to Digital Audio (MUMT203) is a course on DA (200-level) which is part of the Music Science and Technology Minor (MAT) at McGill University. At McGill, courses numbered at the 100 to 400 levels are intended for undergraduate students, and 200-level courses are usually introductory courses for a given discipline.

The course target audience are students from technological and scientific areas who aim to pursue a graduate degree in Music Technology (https://www.mcgill.ca/music/about-us/music-technology) Nevertheless, it is not uncommon for several students with a purely musical background to attend the course every year.

Through almost two decades teaching MUMT203 to students from a variety of academic disciplines and areas, we observed that some of the concepts related to DA are not easily understood by all. For instance, the idea that the sampling process of a time-domain signal inherently generates an infinite number of copies of the signal around multiples of the sampling frequency is not always easy to grasp. In other words, multiplying a time-domain signal (input signal) by an impulse train (sampling signal) creates another impulse train in the frequency domain, with copies of the input signal placed around multiples of the sampling frequency (cf. figure 2, where just the real part of the frequency domain is shown).

During the previous course sections, the instructor and Teaching Assistants (TAs) noted the concepts were understood more easily in dynamic presentations, i.e. observing a particular signal over time, when possible. Over the years, the lecturer and TAs also observed improvements in the learning process when class material included resources such as videos depicting signals over time. ${ }^{4}$

\subsection{Assignments and Class Material}

The DAWb was used in the Fall 2020 section of MUMT203. Given that the class was carried out exclusively online, real-time interactive demonstrations replaced in-person explanations in front of a projector or on a whiteboard, allowing the students anywhere in the world to immediately replicate examples shown by the instructors.

The possibility of presenting different DA scenarios by manipulating the parameters also allowed comparison and facilitated understanding concepts, e.g., gradually increasing the frequency using the slider allows visualizing the effects of input signals approaching Nyquist frequency and its multiples.

The DAWb was also used in assignments for the same course. On the second assignment, one of the questions required the students to use the DAWb to represent a system that receives a sinusoidal signal with frequency $f_{0}=1450 \mathrm{~Hz}$, convert into a discrete signal and back at a continuous signal using sample rate $S_{r}=3 \mathrm{kHz}$. Students were asked to observe the input and output signal representations and report whether the representations were identical or not. If not identical, students were also required to explain how they came to differ. As we can observe in figure 7, the DAWb provided students visual information to analyze the difference between input and reconstructed signals and conclude that the proximity between input and a multiple of the Nyquist frequency creates a phase difference between input and sampling signals, resulting in amplitude fluctuation.

Students were also asked to set specific scenarios using the tool and analyze the differences between input and output signals or describe the behaviour of signals in the frequency domain. These tasks helped understand phenomena otherwise challenging to visualize, e.g., how, in sampling, infinite signal copies are generated in the frequency domain and how these affect signal reconstruction.

\subsection{Feedback and Assessment}

The lecturer and TAs observed an increase in the student's engagement during the term when the DAWb was used in class activities. Students informally provided positive feedback during office hours and reported a deeper understanding of concepts when exploring the DAWb. Thirteen out of fifteen students $(87 \%)$ were able to extract information from the $\mathrm{DAWb}$ views to answer the assignment question described in section 4.1. Students also reported exploring the tool when reviewing class material and often suggested improvements or reported issues.

The ability to visualize situations that are not often explored in textbooks and the unlimited access to the simulations from their homes, contrary to typically limited access to laboratory equipment, were deemed major advantages of using the DAWb in that course.

\footnotetext{
${ }^{4}$ An excellent example of video illustrating these concepts is $D / A$ and $A / D$ / Digital Show and Tell from xiph.org - https://youtu.be/cIQ9IXSUzuM.
} 

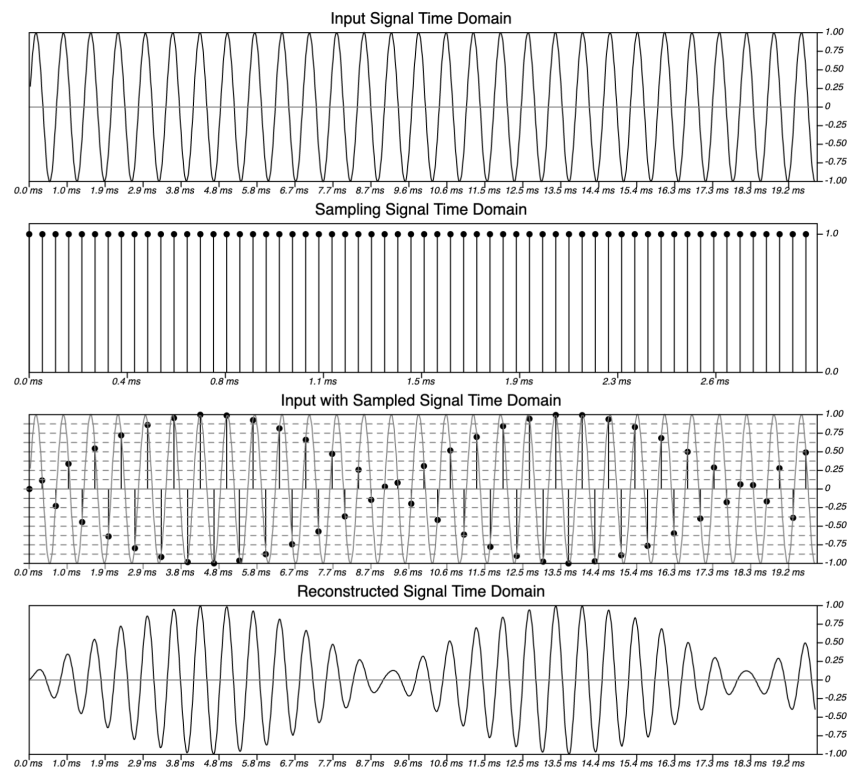

Figure 7: Input, sampling, input overlaid with samples, and reconstructed DAWb time-domain signal panels used for the class assignment.

\section{LIMITATIONS AND FUTURE WORK}

Limitations: One intended limitation of the $\mathrm{DAWb}$ is the discretized scale for sampling frequency due to the choice of using integer submultiples of the maximum sampling rate for resampling (cf. section 3.2.2). This actually comes in handy as it allows for proposing assignment exercises with parameters not directly available in the workbench, so that students can show whether they grasped the concepts without an immediate answer provided by the interactive explanation.

Several non-intended limitations exist, though. The output lowpass filter doesn't perfectly remove images above the Nyquist frequency, something that can be confusing for individuals new to the subject area. Given that the signals are actually processed by the application (and not merely mocked-up), the presence of non-idealities needs to be taken into account. Overall performance should also be improved, allowing for more complex input signals to be used.

Future work: On the technical side, there are a number of useful features we intend to implement in the future. Currently there is no way to store or recall presets, e.g. to demonstrate specific situations or allow students to share their work. We are interested in adding such functionality, perhaps by allowing the state of the simulation to be encoded in a share-able URL with query parameters used to set the parameters of the simulation. While our tool can currently be embedded in a web page using iframes, we are also interested in making the tool easy to embed as a library in web applications, what would facilitate the co-location of interactive examples with text and other pedagogical materials. Another useful feature would be to introduce additional options for the input signal, such as different synthesis mechanisms, allowing live audio input, and/or allowing the user to upload audio files.

\section{CONCLUSIONS}

This paper introduced the IDMIL Digital Audio Workbench, a freely available, open-source interactive platform for exploring concepts in DA theory. The DAWb allows for experimentation with various types of signals and sampling parameters, with immediate simulation of the results in the form of both time- and frequency-domain views of signals as well as audio rendering. Having been extensively used by a cohort of 15 students in an online 13-week 200-level university course on DA, it proved useful for teaching and learning topics such as aliasing, quantization, critical sampling, anti-aliasing filtering and dithering, allowing students to better grasp abstract concepts related to audio signal processing.

\section{ACKNOWLEDGMENTS}

Thanks to Erivan Duarte, Florent Berthaut and James Leonard for suggestions and for P. Charles Reimer for detailed proofreading of an earlier version of this paper. Thanks also to the undergraduate students who used the tool for comments, bug reports and suggestions. This work was funded in part thanks to a Discovery Grant from the Natural Sciences and Engineering Research Council of Canada (NSERC) to the first author.

\section{REFERENCES}

[1] Wendy K. Adams, Sam Reid, Ron Lemaster, Sarah B. McKagan, Katherine K. Perkins, Michael Dubson, and Carl E. Wieman. 2008. A Study of Educational Simulations Part I - Engagement and Learning. Journal of Interactive Learning Research 19, 3 (2008), 397-419.

[2] Jesse Allison, Daniel Holmes, and Zachary Berkowitz. 2016. Programming Music Camp: Using Web Audio to Teach Creative Coding. In Proceedings of the International Web Audio Conference (WAC '16). Georgia Tech, 5.

[3] Mark Ballora. 2017. Digital Audio and Acoustics for the Creative Arts. (1st ed.). Oxford University Press, New York, NY.

[4] Nicky Case. 2021. Explorable Explanations, a Hub for Learning through Play! https://explorables Source: https://github.com/explorableexplanations/explorableexplanations.github.io.

[5] Thierry Dutoit and Ferran Marqués. 2009. Applied Signal Processing. Springer US, Boston, MA.

[6] Christian Frisson, Thomas Pietrzak, Siyan Zhao, and Ali Israr. 2016. WebAudioHaptics: Tutorial on Haptics with Web Audio. In Proceedings of the International Web Audio Conference (WAC'16). Georgia Tech, Atlanta, Georgia, USA.

[7] Kosmas Kritsis, Carlos Acosta, Manuel Bouillon, Robert Piéchaud, Daniel MartínAlbo, and Vassilis Katsouros. 2019. iMuSciCA: A Web Platform for Science Education Through Music Activities. In Proceedings of the International Web Audio Conference (WAC '19). NTNU, 35-40.

[8] James H. McClellan, Ronald W. Schafer, and Mark A. Yoder. 1998. DSP First. A Multimedia Approach. (1st ed.). Prentice Hall, Upper Saddle River, NJ.

[9] Katherine Perkins, Wendy Adams, Michael Dubson, Noah Finkelstein, Sam Reid, Carl E. Wieman, and Ron Lemaster. 2006. PhET: Interactive Simulations for Teaching and Learning Physics. The Physics Teacher 44, 1 (2006), 18.

[10] Ken C. Pohlmann. 2011. Principles of Digital Audio. (sixth ed.). McGraw-Hill, New York, NY

[11] Ken Steiglitz. 1996. A Digital Signal Processing Primer with Applications to Digital Audio and Computer Music (1st ed.). Addison-Wesley, Menlo Park, CA.

[12] Bret Victor. 2011. Explorable Explanations. http://worrydream.com/ ExplorableExplanations/

[13] John Watkinson. 2001. The Art of Digital Audio (3rd ed.). Focal Press, Oxford, $\mathrm{UK}$.

[14] Nils Werner, Stefan Balke, Fabian-Robert Stöter, Meinard Müller, and Bernd Edler. 2017. Trackswitch.Js: A Versatile Web-Based Audio Player for Presenting Scientific Results. In Proceedings of the International Web Audio Conference (WAC '17). Queen Mary University of London, 6.

[15] Carl E. Wieman, Wendy K. Adams, and Katherine K. Perkins. 2008. PhET: Simulations That Enhance Learning. Science 322, 5902 (2008), 682-683.

[16] Anna Xambó, Robin Støckert, Sigurd Saue, and Alexander Refsum Jensenius. 2019. Facilitating Team-Based Programming Learning with Web Audio. In Proceedings of the International Web Audio Conference (WAC '19). NTNU, 2-7. 